\title{
Topotecan Use for Second-Line Treatment in Patients with Recurrent or Metastatic Cervical Cancer at Brazilian National Cancer Institute (INCA) ${ }^{*}$
}

\author{
Leandro Nascimento de Oliveira\#, Flávia Vieira Guerra Alves, Paulo Alexandre Ribeiro Mora, \\ Claudio Calazan do Carmo, Angélica Nogueira-Rodrigues, Alvaro Henrique Ingles Garces ${ }^{\dagger}$, \\ Andréia Cristina de Melo
}

Instituto Nacional do Câncer, Hospital do Câncer II, Rio de Janeiro-RJ, Brazil.
Email: ${ }^{\dagger}$ alvarohenriq@yahoo.com.br

Received March $17^{\text {th }}, 2013$; revised April 20 ${ }^{\text {th }}$, 2013; accepted April 30 ${ }^{\text {th }}, 2013$

Copyright (C) 2013 Leandro Nascimento de Oliveira et al. This is an open access article distributed under the Creative Commons Attribution License, which permits unrestricted use, distribution, and reproduction in any medium, provided the original work is properly cited.

\begin{abstract}
Objective: Cervical cancer represents the third most commonly diagnosed cancer and is an important cause of death for women suffering with malignancies. Patients who are refractory or progressed after first-line palliative treatment have a dismal prognosis and no second-line chemotherapy is considered standard so far. Several agents have been investigated in this setting and topotecan is one of the most characterized. The objective of this study was to evaluate response rate (RR), progression-free survival (PFS), overall survival (OS) and toxicity of topotecan in second palliative line for cervical cancer. Methods: An analysis was performed of all patients with recurrent or metastatic cervical cancer treated with topotecan in second palliative line at Brazilian National Cancer Institute, between 2008 and 2010. Results: A total of 73 courses of topotecan were given in the current study (median: 3.5 cycles; range 1 - 6). Anemia was the most frequent adverse event (grade 2:35\%; grade 3:30\%). Of the 20 patients evaluable, there were 2 partial responders to the treatment. The overall response rate (ORR) was $10 \%$; 3 patients (15\%) had stable disease as maximum response. The median PFS for the entire group was 2.93 months (95\% CI 2.41 - 3.45) and OS was 4.66 months (95\% CI 1.21 - 8.11). Conclusion: The limited activity of topotecan schemas in second-line treatment of cervical cancer and the associated overall toxicity may not justify their use in this setting. Patients who progress after first-line treatment may be offered participation in clinical trials, other second-line agents or best supportive care measures.
\end{abstract}

Keywords: Topotecan; Cervical Cancer; Palliative Treatment

\section{Introduction}

Cervical cancer represents the third most commonly diagnosed cancer [1] and is an important cause of death for women suffering with malignancies; one-third of patients initially treated for early and locally advanced cervical cancer will die due to local or systemic relapse [2]. The 5 -year survival rates for patients with locally advanced or distant cervical cancer are $49 \%$ and $15 \%$ respectively [3] and in accordance to literature the mortality rate in those patients has remained unchanged over the past 25 years [4].

\footnotetext{
*Authors' disclosures of potential conflicts of interest: The authors indicated no potential conflicts of interest.

\#in memorian.

${ }^{\dagger}$ Corresponding author.
}

Palliative systemic chemotherapy, usually including platinum-based regimens, is the best option for patients who have relapsed after primary surgery or cisplatinbased chemoradiation, not amenable for curative treatment, and for those staging IVB at the initial diagnosis; however, the results for the majority of them are disappointing. Patients who are refractory or progressed after first-line palliative treatment have a dismal prognosis with infrequent responses and no second-line chemotherapy is considered standard so far. The treatment usually focuses on symptoms palliation and gains in survival when feasible.

Several agents have been investigated in this setting and topotecan is one of the most characterized. In vitro experiments have shown significant antineoplasic activ- 
ity in squamous cell lines of cervical cancer with topotecan alone as well as an increase in cytotoxicity when used in combination with cisplatin [5].

To date, the use of topotecan as second-line or thirdline therapy in patients with cervical cancer has been studied in five phase II trials.

The first study [6] administered intravenously topotecan at a dose of $1.2 \mathrm{mg} / \mathrm{m}^{2}$ per day during 5 consecutive days, on a 28-day cycle and included 22 patients with squamous cell carcinoma (SCC) or adenocarcinoma who had received prior chemotherapy (1 or 2 regimens). Eighteen patients were evaluated for efficacy with partial response (PR) in $16.6 \%$ and stable disease (SD) in 33.3\% of the patients. All 3 PR cases were diagnosed as SCC.

The second trial, GOG 127-F [7], was restricted to patients with advanced, recurrent or metastatic squamous cell carcinomas; one prior chemotherapy, usually platinum-based, was allowed for metastatic disease. Patients received topotecan at $1.5 \mathrm{mg} / \mathrm{m}^{2}$ per day for 5 days on 21-day cycle. Thirty-four out of 45 patients who were included had received prior chemotherapy. Forty patients were evaluable for response-complete response (CR) in $2.5 \%$, PR in $10 \%$ and SD in $37.5 \%$. The median progression-free interval (PFI) was 2.1 months with a median overall survival (OS) of 6.6 months.

The third study [8] employed topotecan at a regimen of $1.0 \mathrm{mg} / \mathrm{m}^{2}$ per day during 5 consecutive days, every 21 days. Twelve patients with SCC or adenocarcinoma were treated, 7 of them received topotecan as second-line chemotherapy treatment. There were 2 PR (16.7\%).

The fourth phase II [2] trial have demonstrated that an alternative schedule of weekly topotecan at $3.0 \mathrm{mg} / \mathrm{m}^{2}$ (maximum $5.0 \mathrm{mg} /$ dose) administered at days 1, 8 and 15 on 28-day cycles as second or third-line treatment in patients with recurrent or metastatic cervical cancer is safe and well tolerated. Twenty-two patients entered this study and 18 were evaluable for response; no CR or PR were observed and $27.7 \%$ of patients exhibited SD as the best response. Median PFI was 3.5 months and median OS was 7.0 months.

Finally, the fifth was the GOG 127-U trial [4] evaluating weekly topotecan as a single agent in second-line therapy in persistent or recurrent cervical carcinoma. Topotecan was administered at a dose $3.0 \mathrm{mg} / \mathrm{m}^{2}$, repeated every 7 days for 21 days followed by a seven-day recovery, cycles were defined as a 28-day period. Twentyfive patients were evaluable and none responded to the treatment, $40 \%$ had SD. The median progression-free survival (PFS) was 2.4 months.

The main toxicity related to topotecan treatment is myelosuppresion with high incidence of grade 3 and 4 hematologic events and weekly schemes have remarkably proven to ameliorate hematologic toxicity. Nonhe- matologic toxicity is manageable, generally mild, not doselimiting and does not present a dominant pattern.

Herein, the experience of topotecan use for second-line treatment in patients with recurrent or metastatic cervical cancer at Brazilian National Cancer Institute is reported.

\section{Material and Methods}

\subsection{Patient Selection and Data Collection}

This study was approved by the Ethics in Human Research Committee of the Brazilian National Cancer Institute (INCA), Rio de Janeiro, Brazil and conducted in accordance with the Declaration of Helsinki and Good Clinical Practice guidelines.

In order to evaluate RR, PFS, OS and toxicity, an analysis of all cervical cancer patients treated with topotecan in second palliative line at INCA, between 2008 and 2010, was performed. Patients were identified through internal database and must have received one previous chemotherapy line for the treatment of incurable disease. Clinical data including demographics, stage, histology, previous therapies and the toxicity related with topotecan therapy were retrospectively collected by medical records review.

Response was determined by physical examination and predominantly by repeated imaging studies. Response was defined as follows: complete response (CR), partial response (PR), progressive disease (PD) and stable disease (SD). The radiological evaluation was based on the Response Evaluation Criteria in Solid Tumors (RECIST) version 1.0.

Adverse events were supposed to be assessed every cycle using the National Cancer Institute Common Toxicity Criteria (NCI CTCAE), version 3.0.

Before the first cycle of topotecan, patients had a complete medical history and physical examination, documentation of disease progression after or during the first palliative line and laboratory assessment. Prior to each cycle patients had a medical evaluation including adverse events, complete blood count and chemistry panel. Imaging studies were performed in a frequency according to physicians' discretion.

\subsection{Treatment}

A variety of topotecan schedules were prescribed $(0.75$ $\mathrm{mg} / \mathrm{m}^{2}$ per day during 5 consecutive days to $2.0 \mathrm{mg} / \mathrm{m}^{2}$ per day during 3 consecutive days, every 21 days or weekly topotecan at $3.0 \mathrm{mg} / \mathrm{m}^{2}$ administered at days 1 , 8 and 15 on 28-day cycles) in an outpatient setting with cycles repeated until disease progression, prohibitive toxicity or patient refusal for further therapy. Premedication and discharge prescription were in accordance to the guidelines of the Institution. Regimen selection, dose 
adjustments and delays in subsequent cycles were determined by the assistant physician, according to clinical evaluation and presented toxicities. History, physical examination and laboratory evaluations were obtained prior to each treatment cycle.

\subsection{Statistical Analysis}

OS was estimated from time of the first treatment day until death or for living patients, the last available follow-up and PFS was measured from the date of the first palliative chemotherapy infusion to either first progression or death or the date of last contact for patients who are alive and progression-free, in both cases using the Kaplan-Meier method. All analyses were performed with the SPSS software, version 18.0.

\section{Results}

From January 2008 to December 2010, 21 patients were treated at INCA with some topotecan scheme in second-line setting for recurrent or metastatic cervical cancer.

The patient characteristics are described in Table 1. The median age at progression to first palliative line therapy was 50.97 years (range 32 - 63). At diagnosis, 1 patient had early stage, 16 had locally advanced tumors and 4 metastatic disease; the most frequent histology was squamous cell carcinoma (90.5\%). As first approach and with curative intent, the majority of patients (80.9\%) had

Table 1. Patient Characteristics.

\begin{tabular}{|c|c|c|c|}
\hline & & $\mathrm{n}$ & $\%$ \\
\hline \multicolumn{4}{|c|}{ Age (years) } \\
\hline \multicolumn{2}{|c|}{ Median } & \multicolumn{2}{|c|}{50.97} \\
\hline \multicolumn{2}{|c|}{ Range } & \multicolumn{2}{|c|}{$32-63$} \\
\hline \multirow[t]{2}{*}{ Histology } & SCC & 19 & 90.5 \\
\hline & $\mathrm{ADC}$ & 2 & 9.5 \\
\hline \multicolumn{4}{|c|}{ Stage at disease presentation (FIGO) } \\
\hline & IB1 & 1 & 4.7 \\
\hline & IB2, II, III and IVA & 16 & 76.2 \\
\hline & IVB & 4 & 19.1 \\
\hline Prior isolate & & 2 & 9.5 \\
\hline Prior chems & & 15 & 71.4 \\
\hline \multicolumn{4}{|c|}{ Number of cycles } \\
\hline & Median & \multicolumn{2}{|c|}{3.5} \\
\hline & Range & \multicolumn{2}{|c|}{01 (one) - 06 (six) } \\
\hline
\end{tabular}

${ }^{\#}$ At progression to first palliative line therapy. Abbreviations: SCC: squamous cell carcinoma; ADC: adenocarcinoma. received pelvic radiation and $71.4 \%$ concurrent chemo therapy with cisplatin as a radiosensitizing treatment. The first palliative line regimens included most frequently carboplatin and paclitaxel in $90.5 \%$, followed by single agent cisplatin or carboplatin in the rest of the cases. The median number of delivered first line chemotherapy was 6 cycles (range 2 - 8).

Considering one loss of follow-up, a total of 73 courses of topotecan where given in the current study (a median of 3.5 cycles; range 1 - 6).

Taking into acount the possible bias of retrospective assessments and the lack of information regarding toxicities in one treated patient, the main evaluable toxicities which occurred during the entire therapy are summarized in Table 2. Anemia was the most frequent adverse event; grade 2 and 3 anemia were seen in 35\% and 30\% respectively. Hematologic toxicity was not cumulative and patients could receive further cycles of chemotherapy after the initial dose was reduced according to the physician's discretion. There were no treatment-related deaths. Overall, the incidence of non-hematologic toxicity was not serious. Other less common grade 3 events included creatinine elevation in 2 cases and transaminases increasing in one case.

Of the 20 patients evaluable, there were 2 partial responders to the treatment. The overall response rate (ORR) was $10 \%, 3$ patients (15\%) had stable disease as maximum response. Response was not assessed in one patient due to loss of follow-up.

Table 2. Prevalence and grade of adverse effects $(\%)^{*}$.

\begin{tabular}{|c|c|c|c|c|c|}
\hline & & & Grade & & \\
\hline Adverse event & 0 & 1 & 2 & 3 & 4 \\
\hline Anemia & 10.0 & 25.0 & 35.0 & 30.0 & 0.0 \\
\hline Thrombocytopenia & 95.0 & 5.0 & 0.0 & 0.0 & 0.0 \\
\hline Neutropenia & 75.0 & 0.0 & 10.0 & 15.0 & 0.0 \\
\hline Nausea & 55.0 & 20.0 & 15.0 & 10.0 & 0.0 \\
\hline Vomiting & 95.0 & 5.0 & 0.0 & 0.0 & 0.0 \\
\hline Constipation & 70.0 & 15.0 & 15.0 & 0.0 & 0.0 \\
\hline Diarrhea & 90.0 & 5.0 & 0.0 & 5.0 & 0.0 \\
\hline Mucositis & 80.0 & 20.0 & 0.0 & 0.0 & 0.0 \\
\hline Muscle pain & 80.0 & 0.0 & 20.0 & 0.0 & 0.0 \\
\hline Fatigue & 80.0 & 5.0 & 15.0 & 0.0 & 0.0 \\
\hline Anorexia & 95.0 & 0.0 & 5.0 & 0.0 & 0.0 \\
\hline Fever & 90.0 & 10.0 & 0.0 & 0.0 & 0.0 \\
\hline Renal toxicity & 90.0 & 0.0 & 0.0 & 10.0 & 0.0 \\
\hline Alopecia & 75.0 & 25.0 & 0.0 & NA & NA \\
\hline
\end{tabular}

${ }^{*}$ Considering valid information. NA = not applicable. 
The median PFS for the entire group was 2.93 months (95\% CI 2.41 - 3.45) and OS was 4.66 months (95\% CI 1.21 - 8.11).

\section{Discussion}

The combination of paclitaxel and cisplatin (or carboplatin alternatively) is a worldwide current first choice for systemic treatment in advanced and persistent/recurrent cervical cancer not amenable to curative therapy with an overall response rate of $36 \%$ [9]. Patients who have progressed after platinum-based therapy may be treated with second-line schemas, included in clinical studies or receive best supportive care measures.

A variety of second-line agents have been tested in this scenario [10-12] and the efficacy is rather modest. Topotecan is one of the cytotoxic options that had been investigated for antineoplastic activity in a small number of trials. The heterogeneity of patients accrued in these studies render it impossible to compare results among them. It seems that topotecan, as a single agent or in combination, exihibits only modest activity in a population of previously treated patients with cervical cancer albeit at a cost of substantial hematologic toxicity [11].

There is clearly an unmet need for active new therapies in the management of advanced cervical cancer. The poor outcome for these patients warrants the development of novel therapeutic strategies that exploit abnormal tumor biology.

Some targeted drugs modulating different signal transduction pathways are currently under clinical development inhibiting angiogenesis, targeting epidermal growth factor receptor (EGFR), cell cycle, matrix metalloproteinases, cyclooxygenase-2, mammalian target of rapamycin (mTOR) or proteasome, evaluating efficacy and safety [11-13]. However, no phase III trials have been published and consequently no molecularly targeted agents have been approved for use in clinical practice.

The limited activity of topotecan schemas in secondline treatment of cervical cancer and the associated overall toxicity as shown in this retrospective study may not justify their use in this setting. Patients who progress after first-line treatment may be offered participation in clinical trials, other second-line agents or best supportive care measures.

\section{REFERENCES}

[1] J. Ferlay, H. R. Shin, F. Bray, D. Forman, C. Mathers and D. M. Parkinm "GLOBOCAN 2008 v2.0, Cancer Incidence and Mortality Worldwide: IARC Cancer Base No. 10,” International Agency for Research on Cancer, Lyon, 2010. http://globocan.iarc.fr

[2] J. Coronel, L. Cetina, M. Candelaria, A. González-Fierro, D. Arias, D. Cantu, et al., "Weekly Topotecan as Second- or Third-Line Treatment in Patients with Recurrent or Metastatic Cervical Cancer,” Medical Oncology, Vol. 26, No. 2, 2009, pp. 210-214.

doi:10.1007/s12032-008-9108-5

[3] L. A. G. Ries, M. P. Eisner, C. L. Kosary, B. F. Hankey, B. A. Miller, L. Clegg, et al., "SEER Cancer Statistics Review, 1973-1998,” National Cancer Institute, Bethesda, 2012.

http://seer.cancer.gov/Publications/CSR1973_1998/2001

[4] J. V. Fiorica, J. A. Blessing, L. V. Puneky, A. A. Secord, J. S. Hoffman, S. D. Yamada, et al., "A Phase II Evaluation of Weekly Topotecan as a Single Agent Second Line Therapy in Persistent or Recurrent Carcinoma of the Cervix: A Gynecologic Oncology Group Study,” Gynecologic Oncology, Vol. 115, No. 2, 2009, pp. 285-289. doi:10.1016/j.ygyno.2009.07.024

[5] P. Boabang, C. M. Kurbacher, H. Kohlhagen, A. Waida and B. K. Amo-Takyi, "Anti-Neoplastic Activity of Topotecan versus Cisplatin, Etoposide and Paclitaxel in Four Squamous Cell Cancer Cell Lines of the Female Genital Tract Using an ATP-Tumor Chemosensitivity Assay,” Anticancer Drugs, Vol. 11, No. 10, 2000, pp. 843-848.

[6] K. Noda, H. Sasaki, K. Yamamoto, T. Yamamoto, R. Nishimura, T. Sugiyama, et al., "Phase II Trial of Topotecan for Cervical Cancer of the Uterus," Proceedings of the American Society of Clinical Oncologists, Vol. 15, 1996, p. 754.

[7] M. A. Bookman, J. A. Blessing, P. Hanjani, T. J. Herzog and W. A. Andersen, "Topotecan in Squamous Cell Carcinoma of the Cervix: A Phase II Study of the Gynecologic Oncology Group,” Gynecologic Oncology, Vol. 77, No. 3, 2000, pp. 446-449. doi:10.1006/gyno.2000.5807

[8] N. R. Abu-Rustum, S. Lee and L. S. Massad, “Topotecan for Recurrent Cervical Cancer after Platinum-Based Therapy,” International Journal of Gynecological Cancer, Vol. 10, No. 4, 2000, pp. 285-288. doi:10.1046/j.1525-1438.2000.010004285.x

[9] D. H. Moore, J. A. Blessing, R. P. McQuellon, H. T. Thaler, D. Cella, J. Benda, et al., "Phase III Study of Cisplatin with or without Paclitaxel in Stage IVB, Recurrent, or Persistent Squamous Cell Carcinoma of the Cervix: A Gynecologic Oncology Group Study,” Journal of Clinical Oncology, Vol. 22, No. 15, 2004, pp. 3113-3119.

[10] H. J. Long III., "Management of Metastatic Cervical Cancer: Review of the Literature," Journal of Clinical Oncology, Vol. 25, No. 20, 2007, pp. 2966-2974.

[11] F. Zagouri, T. N. Sergentanis, D. Chrysikos, M. Filipits and R. Bartsch, "Molecularly Targeted Therapies in Cervical Cancer. A Systematic Review," Gynecologic Oncology, Vol. 126, No. 2, 2012, pp. 291-303. doi:10.1016/j.ygyno.2012.04.007

[12] B. J. Monk, M. W. Sill, R. A. Burger, H. J. Gray, T. E. Buekers and L. D. Roman, "Phase II Trial of Bevacizumab in the Treatment of Persistent or Recurrent Squamous Cell Carcinoma of the Cervix: A Gynecologic Oncology Group Study,” Journal of Clinical Oncology, Vol. 27, No. 7, 2009, pp. 1069-1074. doi:10.1200/JCO.2008.18.9043 
Cervical Cancer at Brazilian National Cancer Institute (INCA)

[13] A. Nogueira-Rodrigues, C. C. Carmo, C. Viegas, F. Erlich, C. Camisão, K. Fontão, et al., "Phase I Trial of Erlotinib Combined with Cisplatin and Radiotherapy for Pa- tients with Locally Advanced Cervical Squamous Cell Cancer," Clinical Cancer Research, Vol. 14, No. 19, 2008, pp. 6324-6329. doi:10.1158/1078-0432.CCR-07-5112 